\title{
Estimating the Effect of a National Pharmacy-Led Influenza Vaccination Voucher Program on Morbidity, Mortality, and Costs
}

\author{
Tanya Singh, MPH; Michael Taitel, PhD; Dorothy Loy, PharmD, MBA; and Renae Smith-Ray, MA, PhD
}

\begin{abstract}
BACKGROUND: Influenza (also known as "flu") is estimated to cause between 12,000 and 79,000 deaths annually. Vaccinations are beneficial in preventing influenza cases and reducing the likelihood of severe outcomes. Unfortunately, vaccination coverage is low among uninsured populations. Removing the cost barrier can help increase vaccination coverage in this group, averting flu cases and related morbidity and costs.
\end{abstract}

OBJECTIVE: To model the potential effect of providing no-cost flu vaccinations to uninsured individuals on influenza-related morbidity, mortality, and costs.

METHODS: In collaboration with the Department of Health and Human Services and local agencies, Walgreens pharmacies provided free flu vaccinations through a nationwide voucher distribution program. We calculated the redemption rate, potentially averted cases, and estimated cost savings for the 2015-2016 and 2016-2017 flu seasons. Using incidence and vaccine effectiveness estimates from the Centers for Disease Control and Prevention, we calculated the rate of influenza in the general population and the estimated cases averted based on the number of redeemed vouchers. We applied patient age along with parameters from published studies to estimate averted ambulatory care visits, hospitalizations, mortality, productively losses, and overall related costs.

RESULTS: During the 2015-2016 flu season, the pharmacy chain distributed 600,000 vouchers with a redemption rate of $52.3 \%$, resulting in 314,033 flu vaccinations. Improvements were subsequently made to the distribution process to increase utilization rates. There were 400,000 vouchers distributed during the 2016-2017 season with a higher redemption rate of $87.2 \%$, resulting in 348,924 flu vaccinations. The estimated number of potentially averted cases was higher during the 2016-2017 season $(13,347)$ than the 2015-2016 season $(11,537)$ due to a higher redemption rate and increased flu activity. Taken together, we estimated that 8,621 ambulatory care visits, 314 hospitalizations, and 15 deaths were averted due to the flu voucher program. Averted health care costs totaled \$937,494 in ambulatory care visits and $\$ 3,510,055$ in hospitalizations. Averted productivity losses ranged from $\$ 4,473,509$ to $\$ 14,613,502$.

CONCLUSIONS: This study demonstrates the effectiveness of a pharmacyled partnership with local community-based organizations to promote flu vaccinations among uninsured individuals. Our model found that a no-cost flu voucher program has the potential to reduce influenza-related morbidity, mortality, and costs.

J Manag Care Spec Pharm. 2020;26(1):42-47

Copyright $\odot 2020$, Academy of Managed Care Pharmacy. All rights reserved.

\section{What is already known about this subject}

Annual vaccination helps prevent influenza, avert related serious health events, and reduces health care costs.

Vaccination among uninsured individuals is low, largely because of cost.

Pharmacies are convenient locations to obtain flu vaccinations because of location and extended hours.

\section{What this study adds}

This study describes a pharmacy-led collaboration with local community-based organizations to promote immunization through distributing vouchers for no-cost flu vaccinations.

We modeled the effect of such a program on potentially averted influenza cases and found that administering 662,957 free flu vaccinations potentially averted 24,884 cases, 8,621 ambulatory care visits, 314 hospitalizations, 15 deaths, and up to $\$ 19.5$ million in societal cost savings.

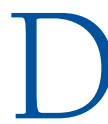
uring the 2017-2018 influenza season, an estimated 960,000 people were hospitalized, and 79,000 people died from influenza (also known as "flu"). ${ }^{1}$ The magnitude of the influenza-related morbidity and mortality poses a notable threat to public health. Although many affected individuals recover without severe complications, seasonal influenza is also responsible for substantial lost productivity and high medical costs. Severe illness, hospitalization, and death occur largely in high-risk populations such as young children, older adults, pregnant women, and people who are immunocompromised. In recent years, the number of symptomatic influenza cases in the United States has ranged from 9.3 to 49 million per season, resulting in 140,000-960,000 hospitalizations and 12,000-79,000 deaths. ${ }^{1}$ Health care costs for influenza-related hospitalizations range from $\$ 5,500$ to $\$ 12,800$ per patient. ${ }^{2}$ Combined medical and productivity losses are estimated to cost society up to $\$ 87.1$ billion annually. ${ }^{3}$

Annual flu vaccinations are recommended each year for everyone aged $\geq 6$ months. The vaccine is typically $40 \%-60 \%$ effective and helps prevent millions of cases of illness and serious complications each year. ${ }^{4}$ Despite the benefits, only $41.1 \%$ of U.S. adults get vaccinated against influenza each year. ${ }^{5}$ Barriers to vaccination include cost, inconvenience, and perception of vaccine ineffectiveness. ${ }^{6}$ For patients with low 
income, cost has been shown to be one of the greatest barriers to vaccination. Individuals with higher immunization copayments generally have lower rates of vaccination. ${ }^{6}$ Before Medicaid expansion, each additional dollar of payment for the flu vaccine was shown to reduce coverage by 1-6 percentage points in Medicaid programs. ${ }^{7}$ The financial burden can be prohibitive for uninsured individuals, who must cover the entire cost of the vaccine. Although Medicaid expansion has worked to cover more of the uninsured, health care access is a significant public health concern for the 24.7 million people who still do not have health insurance. ${ }^{8}$ Just $11.3 \%$ of adults without health insurance get vaccinated against the flu each year, making this group an important target for intervention. ${ }^{6}$ There is great potential to further reduce the number of influenza cases and prevent complications by increasing immunization rates among the uninsured, especially in the $33.2 \%$ who have a chronic condition. ${ }^{9}$

Successful immunization programs work to remove financial barriers, increase access, and use publicity, education, and reminder systems..$^{10}$ In recent years, pharmacies have increased access to influenza immunizations through convenient locations, evening and weekend hours, and walk-in service. ${ }^{11} \mathrm{~A}$ recent study showed that $30.5 \%$ of vaccinations administered in pharmacies were given during the evening, weekends, or holidays. Despite these efforts, for uninsured populations, eliminating the financial burden is essential to increasing flu vaccination coverage. Given their convenient locations and broad reach within communities across the United States, pharmacies are well positioned to work with community-based organizations (CBOs) to promote vaccination in uninsured populations. ${ }^{12,13}$

This study presents findings from one such program that involves the collaboration between a national pharmacy chain and local $\mathrm{CBOs}$ to distribute vouchers for free flu vaccinations. For this intervention, CBOs were identified as local agencies that provided services to members within the surrounding community, particularly those with low income or who were medically underserved. The CBOs identified and conducted outreach to the uninsured populations they served with messages promoting the flu vaccine and information on how to acquire the vaccine from the pharmacy at no charge.

The purpose of this study was to estimate the degree to which providing no-cost influenza immunizations to uninsured individuals could potentially avert influenza cases and related morbidity and costs. We developed an economic model to estimate the potential for a pharmacy-led flu voucher program to reduce influenza-related morbidity, mortality, productivity losses, and health care costs.

\section{Methods}

In 2010, Walgreens Pharmacy began collaborating with the U.S. Department of Health and Human Services to create a flu vaccination voucher program for uninsured patients. The program provides vaccinations at no charge to individuals who have a voucher. Paper vouchers are shipped to pharmacy district managers who work with local CBOs to distribute the vouchers to uninsured individuals. District managers, who typically supervise between 8 and 15 stores, identify organizations that work with the target population and provide the vouchers to them directly to give to their clients. Improvements in voucher distribution were made starting in 2016-2017 in an attempt to increase voucher redemption rates. Beginning in 2016-2017, a higher proportion of vouchers were given to districts that had higher voucher redemption rates compared with districts with relatively lower voucher use.

We conducted a retrospective analysis on patients who redeemed a voucher for the flu vaccine during the 2015-2016 and 2016-2017 influenza seasons. We determined the redemption rate from the number of people who redeemed a voucher to receive a flu vaccination divided by the total number of vouchers distributed during that season. Fixed and seasonspecific data from government and peer-reviewed sources were used to estimate potential averted cases, morbidity, and costs (Table 1). ${ }^{2,3,14-18}$ Costs saved were examined from a societal perspective, since the costs of the program itself were absorbed by the pharmacy chain

Patient age was calculated for use in generating the fixed parameter probabilities and costs. Patients were divided into the following 5 age groups: $7-17,18-49,50-64,65-84$, and 85 years and greater for health care-related estimates. ${ }^{2,3}$ Fixed parameters in the analysis included the probability of ambulatory care by age group $(0.32-0.62)$ at the flat cost of $\$ 108.74,{ }^{14}$ the probability of hospitalization by age group (0.0006-0.0412) and cost ranging from $\$ 5,548.87$ to $\$ 12.885 .44,{ }^{2}$ and the probability of mortality (0.00001-0.0017). ${ }^{3}$ Projected productivity losses were based on median weekly wage earnings for the following age groups, consistent with the Bureau of Labor Statistics (BLS): 18-24, 25-34, 35-44, 45-54, and 55-64 years. ${ }^{15,16}$ The median weekly earnings (WE) were adjusted to reflect the difference between the national median household income and voucher recipients' median block-level household income, $\$ 60,309$ and $\$ 52,382$, respectively (a $14.91 \%$ difference). ${ }^{17}$ Finally, we estimated daily wage by dividing weekly wages by 5 , assuming a typical 40-hour work week. After calculating the daily wages for each age group, the estimated productivity loss per influenza illness were calculated using a low and high range of 1.5 to 4.9 days of work lost. ${ }^{18}$

Low and high range of productivity loss per age group = [(BLS WE for age group XX) $\times 0.8509 \div 5] \times 1.5-$ [(BLS WE for age group XX) $\times 0.8509 \div 5] \times 4.9$

Season-specific parameters incorporated the total U.S. population, the estimated number of influenza cases from the Centers for Disease Control and Prevention (CDC), ${ }^{19,20}$ and 


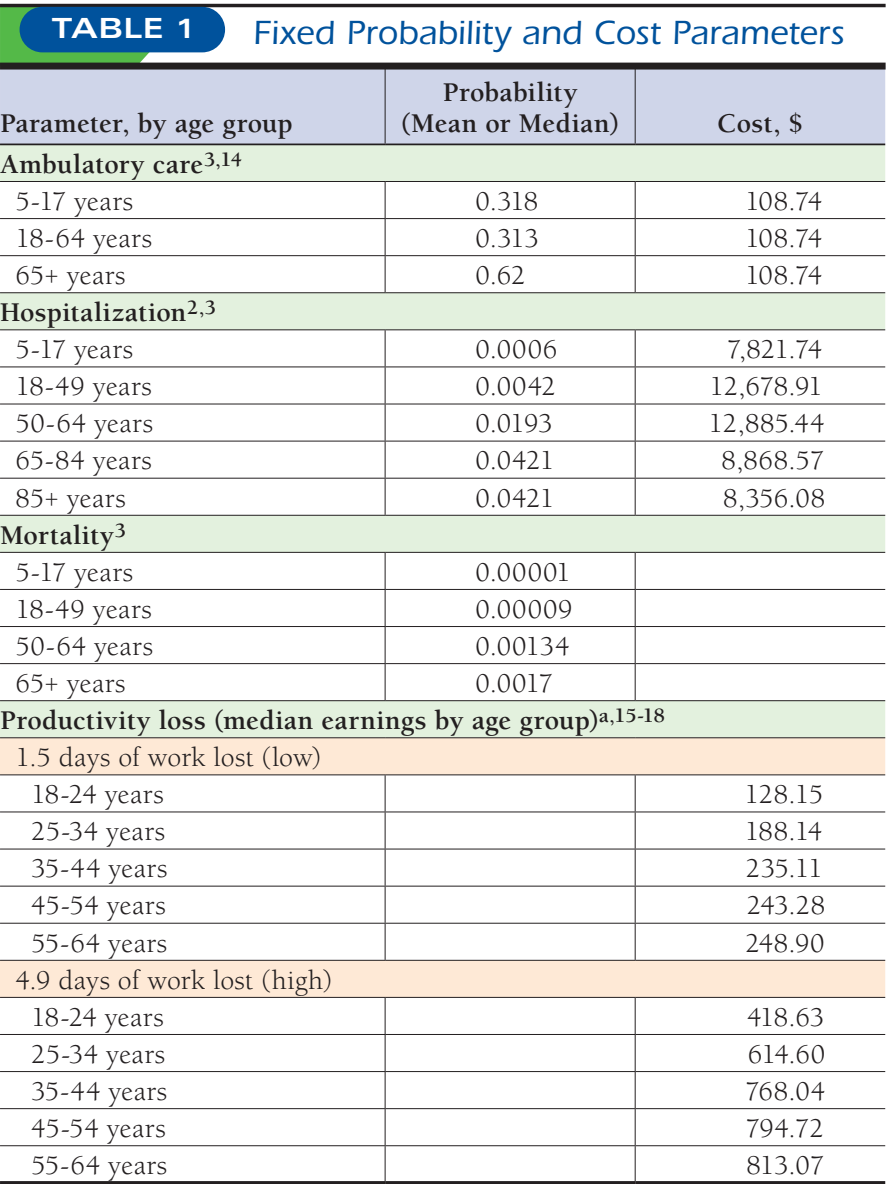

aAdjusted to reflect the difference between the national median household income and voucher recipients' median block-level household income.

vaccine efficacy. ${ }^{21}$ We then determined the influenza attack rate (AR) by dividing the number of influenza cases by the total population.

Estimated influenza $\mathrm{AR}=\mathrm{CDC}$ est. number of influenza cases $\div$ total population

Once the fixed and season-specific parameters were established, the data were applied to the number of patients receiving vaccinations through the voucher program. Potentially averted cases of influenza were calculated by multiplying the number of patients by the attack rate (AR) and the vaccine efficacy (VE).

Potentially averted cases $=$ number of voucher program patients $\times \mathrm{AR} \times \mathrm{VE}$

Next, we used the averted cases (AC) and proportion of voucher recipients in each age group to estimate the ambulatory care visits, hospitalizations, and deaths prevented.
Estimated [visits $\div$ hospitalizations $\div$ deaths] averted per age group $=\mathrm{AC} \times($ prop. voucher recipients in age group $\mathrm{XX}) \times($ prob. of [visit $\div$ hospitalization $\div$ death] in age group XX)

Costs were incorporated to estimate reductions in expenditure for ambulatory care visits, hospitalizations, and productivity losses. Finally, we totaled the health care costs and overall costs averted, including low and high productivity losses.

\section{Results}

One million flu vouchers were distributed during the study period: 600,000 during the 2015-2016 flu season and 400,000 during the 2016-2017 season. The redemption rate increased from $52.3 \%$ in $2015-2016(\mathrm{n}=314,033)$ to $87.2 \%$ in 2016 2017, following implementation of the new distribution plan $(n=348,924)$. Although the number of vouchers distributed was reduced in the second flu season, there was an $11.1 \%$ increase in the number of vouchers redeemed through the program, largely attributable to the tailored distribution of vouchers. Vaccine recipients were from every U.S. state and Puerto Rico. Across the 2 flu seasons, demographic attributes were similar among vaccine recipients. Over both seasons, $58.4 \%$ of vaccine recipients were female; the median age was 45.3 years, with $81.7 \%$ being between 18 and 64 years. Median household income by block-level was $\$ 51,604$ for 2015-2016 and \$51,994 for 2016-2017. Many vaccines (64.6\%) were administered in areas with suburban population size. Rural recipients remained steady at $15.4 \%$ for both flu seasons; however, urban recipients increased from $19.1 \%$ in $2015-2016$ to $20.8 \%$ in $2016-2017$ $(P<0.0001)$.

The efficacy of the 2015-2016 flu vaccine was 48\%, and the total estimated number of cases was 24.6 million. ${ }^{19}$ During this season, our model estimated that the voucher program potentially averted 11,537 influenza cases, between 17,305 and 56,530 lost work days, 3,999 ambulatory care visits, 146 hospitalizations, and 7 deaths. The 2016-2017 season saw a slightly less effective flu vaccine, at $40 \%$ effectiveness, compared with the previous year. The circulating virus was more virulent during this season, resulting in 30.9 million cases of influenza. ${ }^{20}$ Despite the lower vaccine effectiveness, the increase in redeemed vouchers and high number of influenza cases increased the effect of the program. In the 2016-2017 season, the voucher program potentially averted an estimated 13,347 influenza cases, between 20,020 and 65,399 lost work days, 4,622 ambulatory care visits, 168 hospitalizations, and 8 deaths (Table 2)

Estimated health care cost and productivity losses averted were compared for each influenza season. Hospitalization costs made up $78.9 \%$ of total health care costs averted during both seasons. The total health care costs averted for the 2015-2016 flu season was $\$ 2,064,135$ million, with $\$ 434,897$ savings from 


\begin{tabular}{|c|c|c|}
\hline \multicolumn{3}{|c|}{$\begin{array}{l}\text { Computation of Total Cases, Ambulatory } \\
\text { Care, Hospitalizations, and Deaths } \\
\text { Potentially Averted }\end{array}$} \\
\hline Influenza Season & $2015-2016$ & $2016-2017$ \\
\hline Patients who redeemed vouchers, $\mathrm{n}$ & 314,033 & 348,924 \\
\hline \multicolumn{3}{|l|}{ Proportion of patients in each age group } \\
\hline $7-17$ years & 0.072 & 0.078 \\
\hline $18-49$ years & 0.517 & 0.510 \\
\hline $50-64$ years & 0.302 & 0.304 \\
\hline $65-84$ years & 0.098 & 0.097 \\
\hline $85+$ years & 0.011 & 0.010 \\
\hline Estimated number of influenza cases ${ }^{19,20}$ & $24,600,000$ & $30,900,000$ \\
\hline U.S. population ${ }^{19,20}$ & $321,418,800$ & $323,127,500$ \\
\hline $\begin{array}{l}\text { Influenza attack rate } \\
\text { (estimated flu cases } \div \text { total population) }\end{array}$ & 0.077 & 0.096 \\
\hline Vaccine efficacy ${ }^{21}$ & 0.48 & 0.40 \\
\hline $\begin{array}{l}\text { Estimated number of cases if no } \\
\text { vaccination (number of patients who } \\
\text { redeemed vouchers } \times \text { attack rate) }\end{array}$ & 24,035 & 33,367 \\
\hline Total cases averted & 11,537 & 13,347 \\
\hline \multicolumn{3}{|l|}{ Morbidity and mortality averted } \\
\hline Ambulatory care averted & 3,999 & 4,622 \\
\hline Hospitalizations averted & 146 & 168 \\
\hline Deaths averted & 7 & 8 \\
\hline \multicolumn{3}{|l|}{ Costs averted, \$ } \\
\hline Ambulatory costs averted & $434,896.53$ & $502,597.53$ \\
\hline Hospitalizations costs averted & $1,629,239.05$ & $1,880,815.53$ \\
\hline Productivity loss averted (low) & $2,133,090.69$ & $2,467,768.15$ \\
\hline Productivity loss averted (high) & $6,968,096.24$ & $8,061,375.96$ \\
\hline
\end{tabular}

prevented ambulatory visits and \$1,629,239 from prevented hospitalizations. Modeled productivity losses averted ranged from $\$ 2,133,091$ to $\$ 6,968,096$. During the 2016-2017 flu season, the health care costs averted totaled $\$ 2,383,413$, with $\$ 502,598$ saved costs from prevented ambulatory visits and $\$ 1,880,816$ from prevented hospitalizations. Averted productivity losses were between $\$ 2,467,768$ and $\$ 8,061,376$.

\section{Discussion}

After distributing 1 million vouchers over 2 flu seasons, a pharmacy-led vaccine program administered 662,957 free flu vaccinations across the United States and Puerto Rico. Combined, 24,884 cases of influenza, 8,621 ambulatory care visits, 314 hospitalizations, and 15 deaths were potentially prevented. Estimated averted health care costs totaled $\$ 4.5$ million. Taken together with averted productivity losses, up to $\$ 19.5$ million in total societal costs were saved through the program.

In this study, there was an increased number of patients redeeming vouchers for free vaccinations during the second flu season. This occurred despite the decrease in available flu vouchers, demonstrating the effectiveness of the distribution strategy. District managers who experienced a high rate of redeemed vouchers during the 2015-2016 season received a higher proportion of the available vouchers in 2016-2017. This system encouraged district managers to work closely with local $\mathrm{CBOs}$ to ensure that their uninsured clients were targeted for the program. Since this was a pharmacy-led vaccination program versus a traditional vaccination clinic, clients were able to seek out vaccinations at their own convenience without the need to make an appointment.

Regardless of the flu season, demographics show that voucher recipients were not limited to ZIP codes with low income, an indicator of widespread need for no-cost influenza immunizations. Many of the recipients were working-aged adults living in suburban middle-income areas. Providing free flu vouchers to this population bridged the gap in care from those who did not have an employer who offered health insurance or could not otherwise afford insurance but made a high enough income that they did not qualify for Medicaid. Previous studies have found that a third of uninsured patients have a chronic condition, putting them at an increased risk for complications related to influenza. ${ }^{9}$

The cost of providing the free vaccinations was assumed entirely by the pharmacy chain, and the direct benefits were to voucher recipients and local communities. Indirectly, businesses benefit from voucher and similar community programs by building a positive image in the community-a business approach often referred to as "corporate social responsibility." This study demonstrates the potential dual benefit of a pharmacy-led flu voucher program: eliminating the financial barrier to vaccination for uninsured individuals and promoting and increasing vaccination coverage for the benefit of public health.

\section{Limitations}

This study has some limitations to consider. Since the study was a retrospective analysis, we were limited in the demographic information available for each individual vaccine recipient. When individuals came into the pharmacy with vouchers, they were entered as patients into the electronic health record system. The only patient-specific information captured was gender, age, and medication or immunization records. Census-based demographic information was only available from patient ZIP codes or FIPS codes when available.

The design of this study did not allow for randomization or a control group, prohibiting the ability to demonstrate causality. Moreover, if the Walgreens flu vaccination voucher program did not exist, it is possible that voucher recipients would have received flu vaccinations through another source, such as a different program or cash payment for the vaccine, which may have limited the estimated morbidity, mortality, and cost savings estimates attributable to the voucher program. 
We also encountered limitations related to specific fixed parameters. The probability and costs related to ambulatory care and hospitalization were based on the general population, not uninsured populations specifically. For example, it is possible that patients without insurance may be less likely than patients with insurance to seek ambulatory care and recover from influenza without incurring any cost to the health care system. Alternatively, reduced access to primary care may result in an increased number of influenza-related emergency department visits or hospitalization, resulting in higher costs. ${ }^{22-24}$

Also, the estimate for productivity losses was limited to a wide range of days off work caused by seasonal variability in virulence. Again, there may be further considerations for uninsured populations. On one hand, some uninsured patients may be more likely to work while ill because they are likely to suffer financial hardship for missed work compared with insured patients. Other uninsured patients; however, may miss more work if they take longer to recover from influenza due to limited access to primary care and antiviral medication.

\section{Conclusions}

This study found that a no-cost pharmacy-led flu voucher program was effective in promoting flu vaccinations, potentially reducing influenza-related morbidity, mortality, and costs. The program demonstrated a successful collaboration between a chain pharmacy and local CBOs to promote the flu vaccine among uninsured patients and eliminate the cost barrier. Pharmacies are well positioned to promote public health programs, such as vaccination programs, due to their accessibility and reach in local communities.

\section{Authors}

TANYA SINGH, MPH; MICHAEL TAITEL, PhD; DOROTHY LOY, PharmD, MBA; and RENAE SMITH-RAY, MA, PhD, Walgreen Co., Deerfield, Illinois.

AUTHOR CORRESPONDENCE: Tanya Singh, MPH, Walgreen Co., 102 Wilmot Rd., Deerfield, IL 60015. Tel.: 847.964.6520;

E-mail: research@walgreens.com.

\section{DISCLOSURES}

This study was funded by Walgreen Co. All authors are employees of Walgreen Co. and affiliated with Walgreens Center for Health and Wellbeing Research.

Findings from this study were presented as a podium presentation at the Academy of Managed Care Pharmacy Nexus 2018; October 22-25, 2018; Orlando, FL.

\section{ACKNOWLEDGMENTS}

The authors thank Alex Novielli, PharmD, the immunization team, and the Corporate Social Responsibility team for their work on the flu vaccination voucher program and their contributions to the study.

\section{REFERENCES}

1. Centers for Disease Control and Prevention. Past seasons estimated influenza disease burden. 2018. Available at: https://www.cdc. gov/flu/about/ burden/past-seasons.html. Accessed November 29, 2019

2. Agency for Healthcare Research and Quality. HCPnet, Healthcare Cost and Utilization Project. 2018. Available at: https://hcupnet.ahrq.gov. Accessed November 29, 2019.

3. Molinari NA, Ortega-Sanchez IR, Messonnier ML, et al. The annual impact of seasonal influenza in the U.S.: measuring disease burden and costs. Vaccine. 2007;25(27):5086-96

4. Centers for Disease Control and Prevention. Vaccine effectiveness: how well do the flu vaccines work? 2018. Available at: https://www.cdc.gov/flu/ vaccines-work/vaccineeffect.htm. Accessed November 29, 2019.

5. Centers for Disease Control and Prevention. Estimates of Influenza vaccination coverage among adults-United States, 2017-18 flu season. 2018. Available at: https://www.cdc.gov/flu/fluvaxview/coverage-1718estimates. htm. Accessed November 29, 2019.

6. Abbas KM, Kang GJ, Chen D, Werre SR, Marathe A. Demographics, perceptions, and socioeconomic factors affecting influenza vaccination among adults in the United States. PeerJ. 2018;6:e5171

7. Stoecker C, Stewart AM, Lindley MC. The cost of cost-sharing: the impact of Medicaid benefit design on influenza vaccination uptake. Vaccines (Basel). 2017;5(1):8

8. Henry J Kaiser Family Foundation. The number of uninsured people rose in 2017, reversing some of the coverage gains under the Affordable Care Act. December 10, 2018. Available at: https://www.kff.org/uninsured/ press-release/the-number-of-uninsured-people-rose-in-2017-reversing-someof-the-coverage-gains-under-the-affordable-care-act/. Accessed November 29, 2019

9. Christopher AS, McCormick D, Woolhandler S, Himmelstein DU, Bor DH, Wilper AP. Access to care and chronic disease outcomes among Medicaid-insured persons versus the uninsured. Am J Public Health. 2016;106(1):63-69.

10. Williams WW, Lu PJ, O'Halloran A. Surveillance of vaccination coverage among adult populations-United States, 2015. MMWR Surveill Sum. 2017;66(11):1-28.

11. Goad JA, Taitel MS, Fensterheim LE, Cannon AE. Vaccinations administered during off-clinic hours at a national community pharmacy: implications for increasing patient access and convenience. Ann Fam Med. 2013;11(5):429-36.

12. Lindell V, Kelling SE. Review of community based organization and community pharmacy partnerships for preventive care services. INNOVATIONS in Pharmacy. 2018;9(2):Article 8.

13. Ventola CL. Immunization in the United States: recommendations, barriers, and measures to improve compliance: part 2: adult vaccinations. P T. 2016;41(8):492-506

14. Centers for Medicare \& Medicaid Services. Physicians fee schedule. 2017 Available at: https://www.cms.gov/apps/physician-fee-schedule/. Accessed November 29, 2019.

15. Bureau of Labor Statistics. Data retrieval: labor force statistics. 2016. Available at: https://www.bls.gov/webapps/legacy/cpswktab3.htm. Accessed November 29, 2019.

16. Bartsch SM, Taitel MS, DePasse JV, et al. Epidemiologic and economic impact of pharmacies as vaccination locations during an influenza epidemic. Vaccine. 2018;36(46):7054-63. 
17. United States Census Bureau. Income and poverty in the United States. 2016. Available at: https://www.census.gov/data/tables/2017/demo/incomepoverty/p60-259.html. Accessed November 29, 2019.

18. Keech M, Beardsworth P. The impact of influenza on working days lost. Pharmacoeconomics. 2008;26(11):911-24.

19. Centers for Disease Control and Prevention. 2015-2016 estimated influenza illnesses, medical visits, and hospitalizations averted by vaccination in the United States. 2016. Available at: https://www.cdc.gov/flu/about/disease/2015-16.htm. Accessed December 10, 2019.

20. Centers for Disease Control and Prevention. 2016-2017 estimated influenza illnesses, medical visits, and hospitalizations averted by vaccination in the United States. 2017. Available at: https://www.cdc.gov/flu/vaccines-work/ burden-averted-2016-17.htm. Accessed December 10, 2019
21. Centers for Disease Control and Prevention. CDC seasonal flu vaccine effectiveness studies. 2018. Available at: https://www.cdc.gov/flu/vaccineswork/effectiveness-studies.htm. Accessed December 10, 2019.

22. Newton MF, Keirns CC, Cunningham R, Hayward RA, Stanley R. Uninsured adults presenting to US emergency departments: assumptions vs. data. JAMA. 2008;300(16):1914-24.

23. Tammes P, Purdy S, Salisbury C, et al. Can continuity of primary care decrease emergency care use? A nested case-control study. Lancet. 2016;388(Spec Issue):S13 [Abstract]

24. Tsai MH, Xirasagar S, Carroll S, et al. Reducing high-users' visits to the emergency department by a primary care intervention for the uninsured: a retrospective study. Inquiry. 2018;55:46958018763917. 